Research Article

\title{
Nonuniformly Loaded Stack of Antiplane Shear Cracks in One-Dimensional Piezoelectric Quasicrystals
}

\author{
G. E. Tupholme \\ School of Engineering and Informatics, University of Bradford, Bradford BD7 1DP, UK \\ Correspondence should be addressed to G. E. Tupholme; g.e.tupholme@bradford.ac.uk
}

Received 21 May 2018; Revised 18 July 2018; Accepted 2 August 2018; Published 23 September 2018

Academic Editor: Zhiping Luo

Copyright (c) 2018 G. E. Tupholme. This is an open access article distributed under the Creative Commons Attribution License, which permits unrestricted use, distribution, and reproduction in any medium, provided the original work is properly cited.

\begin{abstract}
Representations in a closed form are derived, using an extension to the method of dislocation layers, for the phonon and phason stress and electric displacement components in the deformation of one-dimensional piezoelectric quasicrystals by a nonuniformly loaded stack of parallel antiplane shear cracks. Their dependence upon the polar angle in the region close to the tip of a crack is deduced, and the field intensity factors then follow. These exhibit that the phenomenon of crack shielding is dependent upon the relative spacing of the cracks. The analogous analyses, that have not been given previously, involving nonpiezoelectric or non-quasicrystalline or simply elastic materials can be straightforwardly considered as special cases. Even when the loading is uniform and the crack is embedded in a purely elastic isotropic solid, no explicit representations have been available before for the components of the field at points other than directly ahead of a crack. Typical numerical results are graphically displayed.
\end{abstract}

\section{Introduction}

Since the ground-breaking investigation of strip cracks by Griffith [1], almost a century ago, there has been a flourishing technological need for analyzing the increasingly complex behaviour of defects.

For elastic isotropic solids, Sneddon and Lowengrub [2] obtained the stress-intensity factor in the vicinity of the tip of a strip crack within a row of cracks under the influence of a constant load. Analogously numerous other crack configurations in various materials have been presented. However, invariably most of them do not give representations in a closed form for the field components at a general point, but only directly ahead of the crack.

Here, closed-form expressions are derived for the components of the phonon and phason stresses and electric displacement and field intensity factors created around a stack of parallel shear cracks subjected to nonuniform mechanical and electric loads in onedimensional piezoelectric quasicrystals, using an extension of the method of dislocation arrays. Currently, these are apparently the most comprehensive materials for which it is readily feasible to apply this technique. The corresponding results when the material is simply quasicrystalline or solely piezoelectric or purely isotropic elastic can be deduced as special cases.

The discovery of some unusual complex metallic alloys having quasiperiodic structures was first widely announced by Shechtman et al. [3]. They are now known universally as quasicrystals. The continuing development of the performance and design of smart devices that exploit the effects of piezoelectric coupling of quasicrystals is aided by analyzing the influence of cracks within them.

Experimentally, quasicrystalline materials have been demonstrated to be extremely brittle and often to contain very high densities of multiple cracks. Thus, it is desirable to have a breadth of basic exact solutions readily available for fundamental defect problems, against which more general numerical and experimental studies of analytically intractable practical crack situations can be compared and verified as particular cases. 
The underlying equations that govern the linear elastic theory of quasicrystals within the framework of the mechanics of continua are now widely established. Their solutions continue to be extensively developed for studying a wide variety of boundary value problems and especially those related to cracks. A fundamental analysis that extended the traditional theory of cracks in linear elastic solids to quasicrystals was presented by $\mathrm{Li}$ et al. [4]. Subsequently, Li [5, 6] formulated a general explicit solution of elastohydrodynamic problems in decagonal quasicrystals which enabled wave characteristics in them to be analyzed. Comprehensive accounts and discussions of, and references to, the literature on quasicrystals are conveniently provided for an interested reader by, for example, Ding et al. [7], Guo et al. [8], Fan et al. [9], and Fan $[10,11]$. Yadav [12] gave an interesting summary of the 56 papers that were presented in 2016 at the most recent 13th International Conference on Quasicrystals (ICQ13).

Henceforth, Altay and Dökmeci [13] extended the threedimensional equations of elasticity of quasicrystals to include quasi-static electric effects. This has enabled piezoelectric quasicrystal boundary value problems to be addressed.

The underpinning results of $\mathrm{Li}$ and Liu [14] on the physical property tensors enabled Wang and Pan [15] to give detailed closed-form representations of the field components of a screw dislocation moving uniformly in onedimensional hexagonal piezoelectric quasicrystals. Yang et al. [16] obtained and investigated numerically the electric/elastic field of a straight stationary dislocation based upon the generalized Stroh formalism.

To aid in providing general static solutions to threedimensional boundary value problems in hexagonal piezoelectric quasicrystals, Li et al. [17] applied the rigorous operator theory. Yu et al. [18] and Yu et al. [19] used techniques of complex variable theory for studying a stationary uniformly loaded antiplane Griffith crack and an elliptical cavity, respectively, in a one-dimensional hexagonal piezoelectric quasicrystal.

Within the last two years, the burgeoning depth of research into the effects of piezoelectricity in quasicrystals has continued. The internal and interfacial Green's functions of piezoelectric quasicrystalline bi-materials were obtained by Zhang et al. [20]. Guo and Pan [21] proposed a three-phase cylinder model for composites of piezoelectric quasicrystals. Fundamental solutions for three-dimensional cracks were established by Fan et al. [22] using unit point extended displacement discontinuities and the boundary integral equation method. By means of conformal mappings and complex variables methods, Yang and Li [23] found analytical solutions for a crack emanating from a circular inclusion and Guo et al. [24] studied a composite matrix containing an elliptical inclusion. Both works give the corresponding solutions for a uniformly loaded Griffith crack as special cases. Explicit expressions for the field components of a moving nonconstantly loaded antiplane single crack embedded in an infinite region and within a half-space of one- dimensional hexagonal piezoelectric quasicrystals were determine by Tupholme [25, 26]. Most recently, Fourier transforms and triple integral equations were used by Zhou and $\mathrm{Li}$ [27] to derive solutions in terms of complete elliptical integrals of the first and third kinds for two collinear, constantly loaded, shear cracks in a strip of the piezoelectric quasicrystal.

Within purely piezoelectric media, in which no phason fields are present, many analyses of various crack configurations have been undertaken. It is of note that, in particular, Han and Wang [28] considered a periodic row of parallel, mode I, uniformly loaded cracks situated perpendicular to the surfaces of a piezoelectric strip, by reducing this mixed boundary value problem to the numerical solution of a system of hypersingular integral equations. A periodic distribution of constantly loaded mode III slant cracks was analyzed by Li and Lee [29] using a collocation method and quadrature formula to solve numerically the governing singular integral equations. Zhou et al. [30] studied multiple equally spaced, parallel antiplane shear cracks in infinite piezoelectric materials subjected to constant shear stress loading. Fourier transforms led to dual integral equations which were expanded as a series of Jacobi polynomials and then solved numerically using the Schmidt method. Recently, Monfared and Ayatollahi [31] investigated piezoelectric solids weakened by multiple constantly loaded cracks using Fourier transforms that yielded a set of integral equations for a numerical solution. They provided a useful quite comprehensive review of previous related works of this type.

In Section 2, the general equations in three dimensions that govern deformed piezoelectric quasicrystals are formulated and the physical stack of cracks problem being considered here is stipulated. The relevant constitutive equations for one-dimensional hexagonal piezoelectric quasicrystals with a point group $6 \mathrm{~mm}$ are displayed. The properties of a "piezoelectric quasicrystal screw dislocation" which underpin the current investigation are then outlined in Section 3. A necessary adaptation of the dislocation layer technique is exploited in Section 4 to generate the singular integral equations satisfied by the density functions of the dislocation arrays. These lead to closed-form representations for the resulting phonon, phason, and electric fields and intensity factors created. Their variations with the polar angle near a crack tip are deduced, and graphical numerical results are displayed. In the concluding Section 5, the principal features of this analysis are summarized.

\section{Fundamental Governing Equations of Piezoelectric Quasicrystals and the Stack of Cracks Problem Formulation}

In differential and variationally invariant forms, Altay and Dökmeci [13] presented and studied the governing threedimensional general equations for the deformation components of the phonon, phason, and electric fields within 
a linearized theory of piezoelectric quasicrystalline continua. With repeated suffices $i, j, k, l=1,2,3$ summed according to the traditional Einstein convention, in relation to a rectangular system of fixed Cartesian coordinates $\left(x_{1}, x_{2}, x_{3}\right)$, the appropriate quasi-static equilibrium equations and constitutive equations of such media which are devoid of any applied body forces or electric charge densities can be expressed neatly as follows:

$$
\begin{aligned}
\sigma_{i j, i} & =0, \\
H_{i j, i} & =0 \\
D_{i, i} & =0 \\
\sigma_{i j}= & \frac{c_{i j k l}\left(u_{k, l}+u_{l, k}\right)}{2}+R_{i j k l} w_{k, l}-e_{k i j} E_{k}, \\
H_{i j}= & \frac{R_{k l i j}\left(u_{k, l}+u_{l, k}\right)}{2}+K_{i j k l} w_{k, l}-e_{k i j}^{\prime} E_{k}, \\
D_{i}= & \frac{e_{k i j}\left(u_{j, k}+u_{k, j}\right)}{2}+e_{k i j}^{\prime} w_{j, k}-\varepsilon_{i j} E_{j},
\end{aligned}
$$

where $p$ after a comma, for $p=i, j, k, l$, denotes partial differentiation with respect to $x_{p} . u_{i}$ and $\sigma_{i j}$ are the components of the phonon displacement vector and stress tensor, $w_{i}$ and $H_{i j}$ are the components of the phason displacement vector and stress tensor, and $D_{i}$ and $E_{i}$ are the components of the electric displacement and field. Respectively, $c_{i j k l}, R_{i j k l}, K_{i j k l}, e_{i j k}, e_{i j k}^{\prime}$, and $c_{i j k l}$ are the phonon elastic, the phonon-phason coupling, the phason elastic, the phonon and phason piezoelectric and the dielectric constants.

A one-dimensional hexagonal piezoelectric quasicrystal medium of a point group $6 \mathrm{~mm}$ which is initially in a reference undisturbed stress-free state is studied here. With respect to a fixed Cartesian system of coordinates $(x, y, z)$, the positive $z$-axis coincides with its quasiperiodic direction and it is periodic in the $x-y$ plane.

The material contains an infinite stack of equally spaced, Griffith-type shear cracks parallel to the $x$-axis, each of width $2 a$, that is subjected at infinity to nonuniform phonon, phason, and electrical loads which create a mode III deformation.

The constitutive equations describing the interrelationships between the components $u_{X}, \varepsilon_{X Y}, \sigma_{X Y}$, $w_{X}, w_{z X}, H_{z X}, D_{X}$, and $E_{X}$, respectively, of the phonon displacement vector, phonon strain tensor, phonon stress tensor, phason displacement vector, phason strain tensor, phason stress tensor, electric displacement vector, and electric field vector, for $X$ and $Y=x, y$, or $z$, for deformations of the piezoelectric quasicrystal can be elegantly written in the matrix notation as follows:

$$
\begin{aligned}
& {\left[\begin{array}{l}
\sigma_{x x} \\
\sigma_{y y} \\
\sigma_{z z} \\
\sigma_{y z} \\
\sigma_{x z} \\
\sigma_{x y} \\
H_{z z} \\
H_{z x} \\
H_{z y}
\end{array}\right]=\left[\begin{array}{ccccccccc}
c_{11} & c_{12} & c_{13} & 0 & 0 & 0 & R_{1} & 0 & 0 \\
c_{12} & c_{11} & c_{13} & 0 & 0 & 0 & R_{1} & 0 & 0 \\
c_{13} & c_{13} & c_{33} & 0 & 0 & 0 & R_{2} & 0 & 0 \\
0 & 0 & 0 & 2 c_{44} & 0 & 0 & 0 & 0 & R_{3} \\
0 & 0 & 0 & 0 & 2 c_{44} & 0 & 0 & R_{3} & 0 \\
0 & 0 & 0 & 0 & 0 & c_{11}-c_{12} & 0 & 0 & 0 \\
R_{1} & R_{1} & R_{2} & 0 & 0 & 0 & K_{1} & 0 & 0 \\
0 & 0 & 0 & 0 & 2 R_{3} & 0 & 0 & K_{2} & 0 \\
0 & 0 & 0 & 2 R_{3} & 0 \\
0 & 0 & 0 & K_{2}
\end{array}\right] } \\
& {\left[\begin{array}{l}
\varepsilon_{x x} \\
\varepsilon_{y y} \\
\varepsilon_{z z} \\
\varepsilon_{y z} \\
\varepsilon_{x z} \\
\varepsilon_{x y} \\
w_{z z} \\
w_{z x} \\
w_{z y}
\end{array}\right]-\left[\begin{array}{ccc}
0 & 0 & e_{31} \\
0 & 0 & e_{31} \\
0 & 0 & e_{33} \\
0 & e_{15} & 0 \\
e_{15} & 0 & 0 \\
0 & 0 & 0 \\
0 & 0 & e_{33}^{\prime} \\
e_{15}^{\prime} & 0 & 0 \\
0 & e_{15}^{\prime} & 0
\end{array}\right]\left[\begin{array}{l}
E_{x} \\
E_{y} \\
E_{z}
\end{array}\right], }
\end{aligned}
$$

$$
\begin{aligned}
{\left[\begin{array}{l}
D_{x} \\
D_{y} \\
D_{z}
\end{array}\right]=} & {\left[\begin{array}{ccccccccc}
0 & 0 & 0 & 0 & 2 e_{15} & 0 & 0 & e_{15}^{\prime} & 0 \\
0 & 0 & 0 & 2 e_{15} & 0 & 0 & 0 & 0 & e_{15}^{\prime} \\
e_{31} & e_{31} & e_{33} & 0 & 0 & 0 & e^{\prime}{ }_{33} & 0 & 0
\end{array}\right]\left[\begin{array}{c}
\varepsilon_{x x} \\
\varepsilon_{y y} \\
\varepsilon_{z z} \\
\varepsilon_{y z} \\
\varepsilon_{x z} \\
\varepsilon_{x y} \\
w_{z z} \\
w_{z x} \\
w_{z y}
\end{array}\right] } \\
+ & {\left[\begin{array}{cccc}
\varepsilon_{11} & 0 & 0 \\
0 & \varepsilon_{11} & 0 \\
0 & 0 & \varepsilon_{33}
\end{array}\right]\left[\begin{array}{c}
E_{x} \\
E_{y} \\
E_{z}
\end{array}\right], }
\end{aligned}
$$

with

$$
\begin{aligned}
\varepsilon_{X Y} & =\frac{1}{2}\left(\frac{\partial u_{X}}{\partial Y}+\frac{\partial u_{Y}}{\partial X}\right), \\
w_{z X} & =\frac{\partial w_{z}}{\partial X},
\end{aligned}
$$

Here, the material constants involved have been abbreviated, using the standard contracted notation of Voigt with $i$ and $j$ having integer values, to $c_{i j}$, the phonon elastic moduli, $K_{i}$, the phason elastic moduli, $R_{i}$, the phonon-phason coupling 
elastic moduli, $e_{i j}$ and $e_{i j}^{\prime}$, the piezoelectric moduli, and $\varepsilon_{i j}$, the dielectric moduli.

It is noteworthy to mention at this stage that no previous investigations have been presented deriving explicit expressions for the deformed field components, or their angular variations near the tip of a crack, within a stack of nonuniformly loaded parallel cracks, even in hexagonal piezoelectric crystals or purely isotropic elastic, nor even when the loads are uniform. The study here is for piezoelectric quasicrystals, which are the most general material currently available for which the analysis is amenable. The results can be reduced to those for hexagonal piezoelectric crystals with no phason field involved by throughout putting $R=K=e_{15}^{\prime}=0$ and then those for purely isotropic elastic materials with no piezoelectric effects by also putting $e_{15}=0$, with $c_{44}=\mu$, the second Lame constant.

The cracks are considered to be parallel to each other and a distance $h$ apart, so that they are centred at $x=0$ on the planes $y=n h$ for $n=0, \pm 1, \pm 2, \ldots$, as shown in Figure 1 .

It is convenient to write the electric field vector, $\mathrm{E}$, in terms of an electric potential, $\phi$, so that

$$
\mathbf{E}=-\nabla \phi .
$$

All the field variables in the antiplane deformation which is created are independent of $z$. Thus, from Equation (4), the nonzero phonon and phason strain components are given in terms of the components, $u_{z}$ and $w_{z}$, of the phonon and phason displacements by

$$
\begin{gathered}
\varepsilon_{x z}=\frac{1}{2} \frac{\partial u_{z}}{\partial x}, \\
\varepsilon_{y z}=\frac{1}{2} \frac{\partial u_{z}}{\partial y}, \\
w_{z x}=\frac{\partial w_{z}}{\partial x} \\
w_{z y}=\frac{\partial w_{z}}{\partial y}
\end{gathered}
$$

and, from the constitutive equations (2) and (3), the corresponding nonzero phonon and phason stresses and displacement components are

$$
\begin{aligned}
\sigma_{y z} & =2 c_{44} \varepsilon_{y z}+R w_{z y}-e_{15} E_{y}, \\
\sigma_{x z} & =2 c_{44} \varepsilon_{x z}+R w_{z x}-e_{15} E_{x}, \\
H_{z y} & =2 R \varepsilon_{y z}+K w_{z y}-e_{15}^{\prime} E_{y}, \\
H_{z x} & =2 R \varepsilon_{x z}+K w_{z x}-e_{15}^{\prime} E_{x}, \\
D_{y} & =2 e_{15} \varepsilon_{y z}+e_{15}^{\prime} w_{z y}+\varepsilon_{11} E_{y}, \\
D_{x} & =2 e_{15} \varepsilon_{x z}+e_{15}^{\prime} w_{z x}+\varepsilon_{11} E_{x} .
\end{aligned}
$$

In Equations (7)-(9) and subsequently, for the sake of brevity, the material constants $R_{3}$ and $K_{2}$ are simply written as $R$ and $K$.

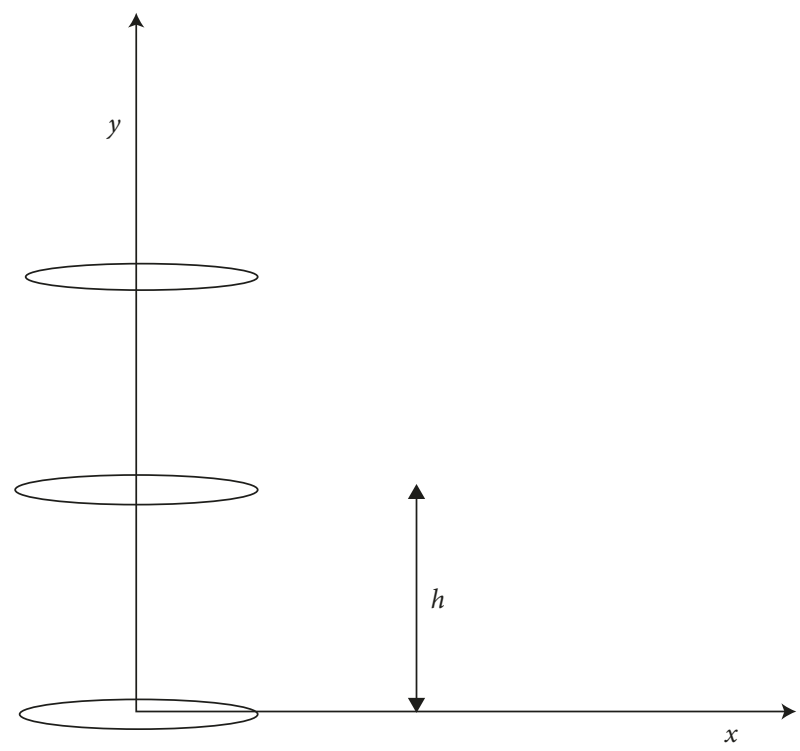

$\mathrm{O}$
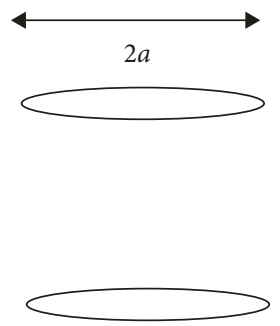

FIGURE 1: Stack of parallel Griffith-type shear cracks.

The nonuniform phonon, phason, and electrical loads, $\mathscr{T}(x), \mathscr{H}(x)$, and $\mathscr{D}(x)$, applied at infinity are symmetrical, so that

$$
\begin{aligned}
\sigma_{y z}(x, y) & \longrightarrow \mathscr{T}(x), \\
H_{z y}(x, y) & \longrightarrow \mathscr{H}(x), \\
D_{y}(x, y) & \longrightarrow \mathscr{D}(x), \text { as } \sqrt{x^{2}+y^{2}} \longrightarrow \infty,
\end{aligned}
$$

with $\mathscr{T}(-x)=\mathscr{T}(x), \mathscr{H}(-x)=\mathscr{H}(x)$, and $\mathscr{D}(-x)=\mathscr{D}(x)$ for all $x$.

Likewise, an interested reader could analyze analogously the mode III deformations which arise by specifying instead any other selection of three of the components $\sigma_{y z}, \varepsilon_{y z}, H_{z y}, w_{z y}, D_{y}$, or $E_{y}$ at infinity.

\section{A Piezoelectric Quasicrystal Screw Dislocation}

It is convenient to first summarize the fundamental features of a "piezoelectric quasicrystal screw dislocation" upon which the subsequent analysis here is founded. This extends to a piezoelectric quasicrystalline medium the traditional notion of a screw dislocation in a linear solely elastic solid material by the introduction of the concept of an extended Burgers vector that has discontinuities in the components, $u_{z}$ and $w_{z}$, of the phonon and phason displacements and 
$\phi$, the electric potential, of magnitudes $b, d$, and $b_{4}$, respectively, across a slip plane.

Wang and Pan [15] presented explicit general expressions for the components of the fields of this type of straight dislocation situated parallel to the $z$-axis at the origin in a one-dimensional hexagonal piezoelectric quasicrystal with a point group $6 \mathrm{~mm}$. From these, it can be deduced that, for such a stationary screw dislocation, the phonon and phason displacement components and the electric potential can be written as

$$
\begin{aligned}
& u_{z}^{\mathrm{III}}(x, y)=\frac{b}{2 \pi} \tan ^{-1}\left(\frac{y}{x}\right), \\
& w_{z}^{\mathrm{III}}(x, y)=\frac{d}{2 \pi} \tan ^{-1}\left(\frac{y}{x}\right), \\
& \varphi^{\mathrm{III}}(\xi, y)=\frac{b_{4}}{2 \pi} \tan ^{-1}\left(\frac{y}{x}\right),
\end{aligned}
$$

where a superscript III is attached to the field variables to indicate that they relate to an antiplane mode III deformation.

For convenience of presentation, it is appropriate to introduce the piezoelectrically stiffened phonon and phason elastic moduli, $\bar{c}_{44}$ and $\bar{K}$, and the phonon-phason coupling elastic constant, $\bar{R}$, as defined in the following equations:

$$
\begin{aligned}
\bar{c}_{44} & =c_{44}+\frac{e_{15}^{2}}{\varepsilon_{11}}, \\
\bar{K} & =K+\frac{e_{15}^{\prime 2}}{\varepsilon_{11}}, \\
\bar{R} & =R+\frac{e_{15} e_{15}^{\prime}}{\varepsilon_{11}} .
\end{aligned}
$$

Recalling the relations (5) and (6), it follows by substituting the expressions (11) into (7)-(9) that the necessary components of the phonon and phason stresses and electric displacement are

$$
\begin{aligned}
\sigma_{x z}^{\mathrm{III}}(x, y) & =-\frac{c_{44} b+R d+e_{15} b_{4}}{2 \pi} \frac{y}{x^{2}+y^{2}}, \\
\sigma_{y z}^{\mathrm{III}}(x, y) & =\frac{c_{44} b+R d+e_{15} b_{4}}{2 \pi} \frac{x}{x^{2}+y^{2}}, \\
H_{z x}^{\mathrm{III}}(x, y) & =-\frac{R b+K d+e_{15}^{\prime} b_{4}}{2 \pi} \frac{y}{x^{2}+y^{2}}, \\
H_{z y}^{\mathrm{III}}(x, y) & =\frac{R b+K d+e_{15}^{\prime} b_{4}}{2 \pi} \frac{x}{x^{2}+y^{2}}, \\
D_{x}^{\mathrm{III}}(x, y) & =-\frac{e_{15} b+e_{15}^{\prime} d-\varepsilon_{11} b_{4}}{2 \pi} \frac{y}{x^{2}+y^{2}}, \\
D_{y}^{\mathrm{III}}(x, y) & =\frac{e_{15} b+e_{15}^{\prime} d-\varepsilon_{11} b_{4}}{2 \pi} \frac{x}{x^{2}+y^{2}} .
\end{aligned}
$$

Likewise, if required, the components of the phonon and phason strain and electric field can be deduced using Equations (6), (11), and (5).

\section{Stack of Shear Cracks Analysis Using an Extension of the Dislocation Layers Technique}

Initially for isotropic elastic solids, the "dislocation layer technique" was devised for modelling loaded strip cracks by equivalent continuous distributions of elastic dislocations which replicate the displacement jump across the crack faces, as usefully described, for example, by Bilby and Eshelby [32] and Lardner [33]. Appropriate arrays of piezoelectric quasicrystal screw dislocations are determined here to extend this concept to the stack of cracks in piezoelectric quasicrystals under consideration.

The screws on the portions to the right of the origin on each crack are positive and those to the left of the origin are negative. The discontinuities in the phonon and phason displacement components and the electric potential must therefore have density functions, $f(x), g(x)$, and $j(x)$, respectively, which are odd functions of $x$.

Because of the symmetry of the problem, the density functions will be identical on each of the cracks, and moreover, it is thus only necessary to ensure that the "central" crack of the stack at $y=0$ is free of phonon and phason stresses and electric displacement.

From Equations (13)-(15), at a general point on the $x$ axis, these dislocation arrays produce phonon and phason stresses and electric displacement having components:

$$
\begin{aligned}
\sigma_{y z}^{\mathrm{III}}(x, 0)= & \frac{b c_{44}}{2 \pi} \int_{-a}^{a} \sum_{n=-\infty}^{\infty} \frac{x-x^{\prime}}{\left(x-x^{\prime}\right)^{2}+n^{2} h^{2}} f\left(x^{\prime}\right) d x^{\prime} \\
& +\frac{d R}{2 \pi} \int_{-a}^{a} \sum_{n=-\infty}^{\infty} \frac{x-x^{\prime}}{\left(x-x^{\prime}\right)^{2}+n^{2} h^{2}} g\left(x^{\prime}\right) d x^{\prime} \\
& +\frac{b_{4} e_{15}}{2 \pi} \int_{-a}^{a} \sum_{n=-\infty}^{\infty} \frac{x-x^{\prime}}{\left(x-x^{\prime}\right)^{2}+n^{2} h^{2}} j\left(x^{\prime}\right) d x^{\prime},
\end{aligned}
$$

$$
\begin{aligned}
H_{z y}^{\mathrm{III}}(x, 0)= & \frac{b R}{2 \pi} \int_{-a}^{a} \sum_{n=-\infty}^{\infty} \frac{x-x^{\prime}}{\left(x-x^{\prime}\right)^{2}+n^{2} h^{2}} f\left(x^{\prime}\right) d x^{\prime} \\
& +\frac{d K}{2 \pi} \int_{-a}^{a} \sum_{n=-\infty}^{\infty} \frac{x-x^{\prime}}{\left(x-x^{\prime}\right)^{2}+n^{2} h^{2}} g\left(x^{\prime}\right) d x^{\prime} \\
& +\frac{b_{4} e^{\prime}}{2 \pi} \int_{-a}^{a} \sum_{n=-\infty}^{\infty} \frac{x-x^{\prime}}{\left(x-x^{\prime}\right)^{2}+n^{2} h^{2}} j\left(x^{\prime}\right) d x^{\prime},
\end{aligned}
$$

$$
\begin{aligned}
D_{y}^{\mathrm{III}}(x, 0)= & \frac{b e_{15}}{2 \pi} \int_{-a}^{a} \sum_{n=-\infty}^{\infty} \frac{x-x^{\prime}}{\left(x-x^{\prime}\right)^{2}+n^{2} h^{2}} f\left(x^{\prime}\right) d x^{\prime} \\
& +\frac{d e^{\prime} 15}{2 \pi} \int_{-a}^{a} \sum_{n=-\infty}^{\infty} \frac{x-x^{\prime}}{\left(x-x^{\prime}\right)^{2}+n^{2} h^{2}} g\left(x^{\prime}\right) d x^{\prime} \\
& -\frac{b_{4} \varepsilon_{11}}{2 \pi} \int_{-a}^{a} \sum_{n=-\infty}^{\infty} \frac{x-x^{\prime}}{\left(x-x^{\prime}\right)^{2}+n^{2} h^{2}} j\left(x^{\prime}\right) d x^{\prime} .
\end{aligned}
$$


The equations of equilibrium that necessarily must be fulfilled to satisfy the imposed boundary conditions (10) are that

$$
\begin{aligned}
\sigma_{y z}^{\mathrm{III}}(x, 0) & =-\mathscr{T}(x), \\
H_{z y}^{\mathrm{III}}(x, 0) & =-\mathscr{H}(x), \\
D_{y}^{\mathrm{III}}(x, 0) & =-\mathscr{D}(x), \quad \text { for }|x|<a .
\end{aligned}
$$

By substituting the expressions (16)-(18) into the conditions (19), a system of three coupled simultaneous equations is obtained. After suitable algebraic manipulation, its solutions lead to three singular integral equations to be satisfied by the density functions, $f(\xi), g(\xi)$, and $j(\xi)$, in the following forms:

$$
\begin{aligned}
& \int_{-a}^{a} \sum_{n=-\infty}^{\infty} \frac{x-x^{\prime}}{\left(x-x^{\prime}\right)^{2}+n^{2} h^{2}} f\left(x^{\prime}\right) d x^{\prime} \\
& =-\frac{2 \pi}{b \varepsilon_{11}\left(\bar{c}_{44} \bar{K}-\bar{R}^{2}\right)} \times\left\{\varepsilon_{11} \bar{K} \mathscr{T}(x)-\varepsilon_{11} \bar{R} \mathscr{H}(x)\right. \\
& \left.\quad-\left(e_{15}^{\prime} \bar{R}-e_{15} \bar{K}\right) \mathscr{D}(x)\right\}, \\
& \int_{-a}^{a} \sum_{n=-\infty}^{\infty} \frac{x-x^{\prime}}{\left(x-x^{\prime}\right)^{2}+n^{2} h^{2}} g\left(x^{\prime}\right) d x^{\prime} \\
& =-\frac{2 \pi}{d \varepsilon_{11}\left(\bar{c}_{44} \bar{K}-\bar{R}^{2}\right)} \times\left\{-\varepsilon_{11} \bar{R} \mathscr{T}(x)+\varepsilon_{11} \bar{c}_{44} \mathscr{H}(x)\right. \\
& \left.\quad+\left(e_{15}^{\prime} \bar{c}_{44}-e_{15} \bar{R}\right) \mathscr{D}(x)\right\},
\end{aligned}
$$

$$
\begin{aligned}
\int_{-a}^{a} \sum_{n=-\infty}^{\infty} \frac{x-x^{\prime}}{\left(x-x^{\prime}\right)^{2}+n^{2} h^{2}} j\left(x^{\prime}\right) d x^{\prime} \\
=-\frac{2 \pi}{b_{4} \varepsilon_{11}\left(\bar{c}_{44} \bar{K}-\bar{R}^{2}\right)} \times\left\{-\left(e^{\prime}{ }_{15} \bar{R}-e_{15} \bar{K}\right) \mathscr{T}(x)\right. \\
\left.\quad+\left(e^{\prime}{ }_{15} \bar{c}_{44}-e_{15} \bar{R}\right) \mathscr{H}(x)+\left(c_{44} K-R^{2}\right) \mathscr{D}(x)\right\} .
\end{aligned}
$$

The combined contributions to the integrands in Equations (20)-(22) from each of the individual cracks in the stack, as represented by the sum of the doubly-infinite series, can be neatly evaluated using the identity

$$
\sum_{n=-\infty}^{\infty} \frac{z}{z^{2}+n^{2} h^{2}}=\frac{\pi}{h} \operatorname{coth}\left(\frac{\pi z}{h}\right),
$$

and the property that the density functions are odd functions. This enables the left-hand side of Equation (20), for example, to be rewritten as

$$
\frac{\pi}{h} \int_{-a}^{a} \frac{\cosh \left(\pi x^{\prime} / h\right)}{\sinh (\pi x / h)-\sinh \left(\pi x^{\prime} / h\right)} f\left(x^{\prime}\right) d x^{\prime} .
$$

With the introduction of new variables given by

$$
\begin{aligned}
& x_{2}=\sinh \left(\frac{\pi x}{h}\right), \\
& a_{2}=\sinh \left(\frac{\pi a}{h}\right), \\
& x_{2}^{\prime}=\sinh \left(\frac{\pi x^{\prime}}{h}\right),
\end{aligned}
$$

the integral equation (20) for $f(x)$ thus becomes

$$
\begin{aligned}
\int_{-a_{2}}^{a_{2}} \frac{f_{2}\left(x_{2}^{\prime}\right)}{x_{2}-x_{2}^{\prime}} d x_{2}^{\prime} \\
=-\frac{2 \pi}{b \varepsilon_{11}\left(\bar{c}_{44} \bar{K}-\bar{R}^{2}\right)} \times\left\{\varepsilon_{11} \bar{K} \mathscr{T}(x)-\varepsilon_{11} \bar{R} \mathscr{H}(x)\right. \\
\left.\quad-\left(e_{15}^{\prime} \bar{R}-e_{15} \bar{K}\right) \mathscr{D}(x)\right\}, \text { for }\left|x_{2}\right|<a_{2},
\end{aligned}
$$

where $f_{2}\left(x_{2}\right)=f_{2}(\sinh (\pi x / h)) \equiv f(x)$. In the interpretation of this improper integral, its Cauchy principal value is needed in accordance with the Plemelj formulae. Classical techniques, as expounded by, for example, Muskhelishvili [34] and Gakhov [35], readily yield its suitable solution in the form

$$
\begin{aligned}
f_{2}\left(x_{2}\right)= & -\frac{2}{\pi b \varepsilon_{11}\left(\bar{c}_{44} \bar{K}-\bar{R}^{2}\right)} \frac{1}{\left(a_{2}^{2}-x_{2}^{2}\right)^{1 / 2}} \\
& \cdot \int_{-a_{2}}^{a_{2}} \frac{\left(a_{2}^{2}-x_{2}^{\prime 2}\right)^{1 / 2}}{x_{2}^{\prime}-x_{2}} \times\left\{\varepsilon_{11} \bar{K} \mathscr{T}\left(x_{2}^{\prime}\right)-\varepsilon_{11} \bar{R} \mathscr{H}\left(x_{2}^{\prime}\right)\right. \\
& \left.-\left(e_{15}^{\prime} \bar{R}-e_{15} \bar{K}\right) \mathscr{D}\left(x_{2}^{\prime}\right)\right\} d x_{2}^{\prime} .
\end{aligned}
$$

Likewise, it is found that the phason and electric densities that fulfill Equations (21) and (22) are

$$
\begin{aligned}
g_{2}\left(x_{2}\right)= & -\frac{2}{\pi d \varepsilon_{11}\left(\bar{c}_{44} \bar{K}-\bar{R}^{2}\right)} \frac{1}{\left(a_{2}^{2}-x_{2}^{2}\right)^{1 / 2}} \\
& \cdot \int_{-a_{2}}^{a_{2}} \frac{\left(a_{2}^{2}-x_{2}^{\prime 2}\right)^{1 / 2}}{x_{2}^{\prime}-x_{2}} \times\left\{-\varepsilon_{11} \bar{R} \mathscr{T}\left(x_{2}^{\prime}\right)\right. \\
& \left.+\varepsilon_{11} \bar{c}_{44} \mathscr{H}\left(x_{2}^{\prime}\right)+\left(e_{15}^{\prime} \bar{c}_{44}-e_{15} \bar{R}\right) \mathscr{D}\left(x_{2}^{\prime}\right)\right\} d x_{2}^{\prime},
\end{aligned}
$$

$$
\begin{aligned}
j_{2}\left(x_{2}\right)= & -\frac{2}{\pi b_{4} \varepsilon_{11}\left(\bar{c}_{44} \bar{K}-\bar{R}^{2}\right)} \frac{1}{\left(a_{2}^{2}-x_{2}^{2}\right)^{1 / 2}} \int_{-a_{2}}^{a_{2}} \frac{\left(a_{2}^{2}-x_{2}^{\prime 2}\right)^{1 / 2}}{x_{2}^{\prime}-x_{2}} \\
& \times\left\{-\left(e_{15}^{\prime} \bar{R}-e_{15} \bar{K}\right) \mathscr{T}\left(x_{2}^{\prime}\right)+\left(e_{15}^{\prime} \bar{c}_{44}-e_{15} \bar{R}\right) \mathscr{H}\left(x_{2}^{\prime}\right)\right. \\
& \left.+\left(c_{44} K-R^{2}\right) \mathscr{D}\left(x_{2}^{\prime}\right)\right\} d x_{2}^{\prime},
\end{aligned}
$$

where $g_{2}\left(x_{2}\right)=g_{2}(\sinh (\pi x / h)) \equiv g(x)$ and $j_{2}\left(x_{2}\right)=$ $j_{2}(\sinh (\pi x / h)) \equiv j(x)$. It is of note that, generally, these above density functions all depend upon the loads $\mathscr{T}(x)$, 
$\mathscr{H}(x)$, and $\mathscr{D}(x)$ that are applied, the values of the geometric constants $a$ and $h$, and the data for the piezoelectric quasicrystal moduli.

Having determined representations for the densities required for the boundary conditions to be satisfied, closedform expressions can be derived from Equations (6), (10), (11), (13)-(15), and (27)-(29) for all the phonon, phason, and electric field components of interest.

To illustrate the kind of analysis which is involved, it can be seen from Equations (10) and (13), for example, that

$$
\begin{aligned}
\sigma_{y z}(x, y)= & \mathscr{T}(x)+\frac{b c_{44}}{2 \pi} \int_{-a}^{a} \sum_{n=-\infty}^{\infty} \frac{x-x^{\prime \prime}}{\left(x-x^{\prime \prime}\right)^{2}+(y-n h)^{2}} f\left(x^{\prime \prime}\right) d x^{\prime \prime} \\
& +\frac{d R}{2 \pi} \int_{-a}^{a} \sum_{n=-\infty}^{\infty} \frac{x-x^{\prime \prime}}{\left(x-x^{\prime \prime}\right)^{2}+(y-n h)^{2}} g\left(x^{\prime \prime}\right) d x^{\prime \prime} \\
& +\frac{b_{4} e_{15}}{2 \pi} \int_{-a}^{a} \sum_{n=-\infty}^{\infty} \frac{x-x^{\prime \prime}}{\left(x-x^{\prime \prime}\right)^{2}+(y-n h)^{2}} j\left(x^{\prime \prime}\right) d x^{\prime \prime},
\end{aligned}
$$

$$
\begin{aligned}
\sigma_{x z}(x, y)= & -\frac{b c_{44}}{2 \pi} \int_{-a}^{a} \sum_{n=-\infty}^{\infty} \frac{y-n h}{\left(x-x^{\prime \prime}\right)^{2}+(y-n h)^{2}} f\left(x^{\prime \prime}\right) d x^{\prime \prime} \\
& -\frac{d R}{2 \pi} \int_{-a}^{a} \sum_{n=-\infty}^{\infty} \frac{y-n h}{\left(x-x^{\prime \prime}\right)^{2}+(y-n h)^{2}} g\left(x^{\prime \prime}\right) d x^{\prime \prime} \\
& -\frac{b_{4} e_{15}}{2 \pi} \int_{-a}^{a} \sum_{n=-\infty}^{\infty} \frac{y-n h}{\left(x-x^{\prime \prime}\right)^{2}+(y-n h)^{2}} j\left(x^{\prime \prime}\right) d x^{\prime \prime} .
\end{aligned}
$$

At first sight, substitution directly of the expressions for the densities from Equations (27)-(29) into the above leads to very unwieldy double integral representations for these components of stress. But in fact, as summarized in Equations (A.1)-(A.11) of the appendix, after the $x^{\prime \prime}$ integration has been evaluated first and much rearrangement and cancellation algebraically, it is found that concisely

$$
\begin{aligned}
& \sigma_{y z}(x, y)=\mathscr{T}(x)+\mathscr{F}^{\mathscr{T}}(\theta), \\
& \sigma_{x z}(x, y)=-\mathscr{F}^{\mathscr{T}}\left(\theta-\frac{\pi}{2}\right),
\end{aligned}
$$

where the functions $\mathscr{F}^{F}(\theta), \mathscr{R}(x, y)$, and $\theta(x, y)$ are defined for $F(x)=\mathscr{T}(x), \mathscr{H}(x)$, and $\mathscr{D}(x)$ by

$$
\begin{gathered}
\mathscr{F}^{F}(\theta)=-\frac{1}{\pi \mathscr{R}} \int_{-a_{2}}^{a_{2}} \frac{\mathscr{M}_{1}\left(x, x_{2}^{\prime}, \theta\right)}{\mathscr{M}_{2}\left(x, x_{2}^{\prime}\right)}\left(a_{2}^{2}-x_{2}^{\prime 2}\right)^{1 / 2} F\left(x_{2}^{\prime}\right) d x_{2}^{\prime}, \\
\mathscr{R} e^{i \theta}=\left[a_{2}^{2}-\sinh ^{2}\left\{\frac{\pi(x+i y)}{h}\right\}\right]^{1 / 2}
\end{gathered}
$$

with

$$
\begin{aligned}
\mathscr{M}_{1}\left(x, x_{2}^{\prime}, \theta\right)= & \left\{\sinh \left(\frac{\pi x}{h}\right) \cos \left(\frac{\pi y}{h}\right)-x_{2}^{\prime}\right\} \sin \theta \\
& +\cosh \left(\frac{\pi x}{h}\right) \sin \left(\frac{\pi y}{h}\right) \cos \theta, \\
\mathscr{M}_{2}\left(x, x_{2}^{\prime}\right)= & \left\{\sinh \left(\frac{\pi x}{h}\right) \cos \left(\frac{\pi y}{h}\right)-x_{2}^{\prime}\right\}^{2} \\
& +\cosh ^{2}\left(\frac{\pi x}{h}\right) \sin ^{2}\left(\frac{\pi y}{h}\right) .
\end{aligned}
$$

The branches of the square root function in Equation (34) are chosen to specify that $\theta$ is zero for $-a<x<a$ and $y=0+$ and extended elsewhere by analytic continuation.

Similarly utilizing Equations (14) and (15) yields

$$
\begin{aligned}
& H_{z y}(x, y)=\mathscr{H}(x)+\mathscr{F}^{\mathscr{H}}(\theta), \\
& H_{z x}(x, y)=-\mathscr{F}^{\mathscr{H}}\left(\theta-\frac{\pi}{2}\right), \\
& D_{y}(x, y)=\mathscr{D}(x)+\mathscr{F}^{\mathscr{D}}(\theta), \\
& D_{x}(x, y)=-\mathscr{F}^{\mathscr{D}}\left(\theta-\frac{\pi}{2}\right) .
\end{aligned}
$$

With the imposed boundary conditions (10), it can be seen from Equations (32)-(38) and recalling Equation (25) that the phonon stress components, phason stress components, and the electric displacement components individually are decoupled and depend, respectively, upon the applied loads, $\mathscr{T}(x), \mathscr{H}(x)$, and $\mathscr{D}(x)$, but that they are all dependent upon the geometric parameters $a$ and $h$.

Close to a crack tip, the practically interesting variation of these field components with the polar angle can be studied by defining polar coordinates $r$ and $\psi$, with $r \ll a$, so that

$$
\begin{aligned}
& x=a+r \cos \psi, \\
& y=r \sin \psi .
\end{aligned}
$$

Then, as $r \longrightarrow 0$, Equation (34) shows that approximately

$$
\begin{aligned}
\mathscr{R} & \sim\left\{r \frac{2 \pi}{h} \sinh \left(\frac{\pi a}{h}\right) \cosh \left(\frac{\pi a}{h}\right)\right\}^{1 / 2}, \\
\theta & \sim-\left(\frac{\pi-\psi}{2}\right),
\end{aligned}
$$

and correspondingly, Equations (32)-(38) yield 


$$
\begin{aligned}
\sigma_{y z}(r, \psi) & \sim \frac{K_{\mathscr{T}}^{S}}{\sqrt{r}} \cos \left(\frac{\psi}{2}\right), \\
H_{z y}(r, \psi) & \sim \frac{K_{\mathscr{H}}^{S}}{\sqrt{r}} \cos \left(\frac{\psi}{2}\right), \\
D_{y}(r, \psi) & \sim \frac{K_{\mathscr{D}}^{S}}{\sqrt{r}} \cos \left(\frac{\psi}{2}\right), \\
\sigma_{x z}(r, \psi) & \sim-\frac{K_{\mathscr{T}}^{S}}{\sqrt{r}} \sin \left(\frac{\psi}{2}\right), \\
H_{z x}(r, \psi) & \sim-\frac{K_{\mathscr{H}}^{S}}{\sqrt{r}} \sin \left(\frac{\psi}{2}\right), \\
D_{x}(r, \psi) & \sim-\frac{K_{\mathscr{D}}^{S}}{\sqrt{r}} \sin \left(\frac{\psi}{2}\right),
\end{aligned}
$$

where the phonon and phason stress and electric displacement intensity factors, $K_{\mathscr{T}}^{S}, K_{\mathscr{H}}^{S}$, and $K_{\mathscr{D}}^{S}$, at the tip of a crack within a stack of cracks, are defined for $F(x)=\mathscr{T}(x), \mathscr{H}(x)$, and $\mathscr{D}(x)$ by

$$
\begin{aligned}
K_{F}^{S}= & \left\{\frac{1}{2 \pi h \sinh (\pi a / h) \cosh (\pi a / h)}\right\}^{1 / 2} \\
& \cdot \int_{-a}^{a}\left\{\frac{\sinh (\pi a / h)+\sinh \left(\pi x^{\prime} / h\right)}{\sinh (\pi a / h)-\sinh \left(\pi x^{\prime} / h\right)}\right\}^{1 / 2} \\
& \cdot \cosh \left(\frac{\pi x^{\prime}}{h}\right) F\left(x^{\prime}\right) d x^{\prime} .
\end{aligned}
$$

It can be observed that $K_{F}^{S} \longrightarrow K_{F}$ in the limit as $h \longrightarrow \infty$, where

$$
K_{F}=\frac{1}{\pi \sqrt{2 a}} \int_{-a}^{a}\left(\frac{a+x^{\prime}}{a-x^{\prime}}\right)^{1 / 2} F\left(x^{\prime}\right) d x^{\prime},
$$

which is in agreement with the intensity factors derived by Tupholme [25] for a single, nonuniformly loaded Griffithtype shear crack.

Furthermore, the polar components of the phonon stress, phason stress, and electric displacement, $\sigma_{\psi z}, H_{z \psi}$, and $D_{\psi}$, respectively, near a crack tip are deduced from Equations (41) and (42) to be

$$
\begin{aligned}
\sigma_{\psi z}(r, \psi) & \sim \frac{K_{\mathscr{T}}^{S}}{\sqrt{r}} \cos \left(\frac{\psi}{2}\right), \\
H_{z \psi}(r, \psi) & \sim \frac{K_{\mathscr{H}}^{S}}{\sqrt{r}} \cos \left(\frac{\psi}{2}\right), \\
D_{\psi}(r, \psi) & \sim \frac{K_{\mathscr{D}}^{S}}{\sqrt{r}} \cos \left(\frac{\psi}{2}\right),
\end{aligned}
$$

with their variation with the nonuniform loads contained entirely in the intensity factors given by Equation (43).

The distribution around a crack tip with the angle $\psi$ of the scaled phonon stress component, $\sqrt{r} \sigma_{\psi z} / K_{\mathscr{T}}^{S}$, as evaluated from Equation (45), is depicted in Figure 2.

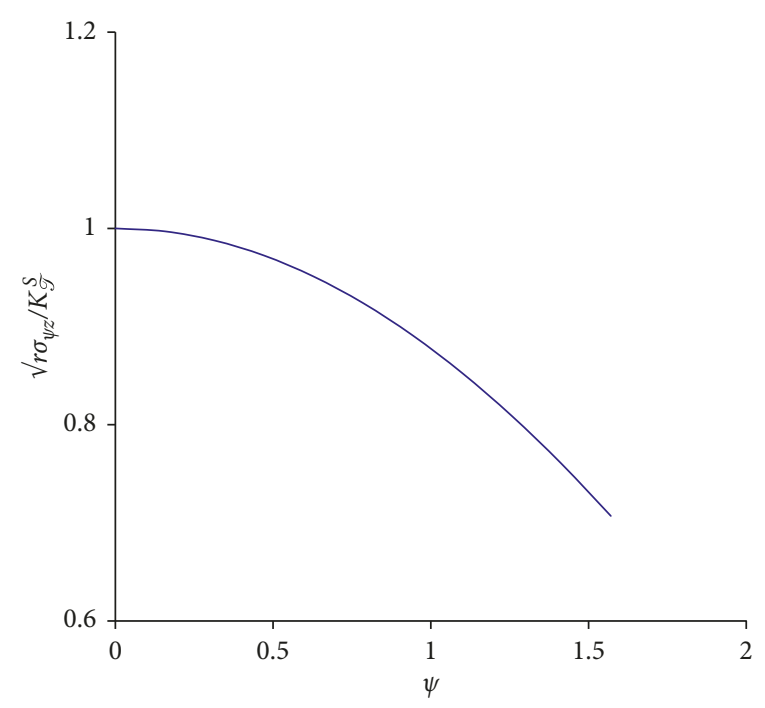

FIGURE 2: Variation of the scaled phonon stress component, $\sqrt{r} \sigma_{\psi z} / K_{\mathscr{T}}^{S}$, around a crack tip.

Clearly from the above, the phonon and phason stress and electric displacement components exhibit the same $1 / \sqrt{r}$ crack-tip behaviour as that close to the tip of a loaded crack embedded in a classical purely elastic isotropic medium.

It is also observed from Equations (27)-(29) that all the density functions are dependent not only upon the geometrical parameters $a$ and $h$ and the imposed loads $\mathscr{T}(x)$, $\mathscr{H}(x)$, and $\mathscr{D}(x)$ but also upon the piezoelectric quasicrystal material constants. But on the other hand, it is seen from Equations (41)-(43) and (45) that the components of the phonon stress, phason stress, and electric displacement are decoupled and that they and the field intensity factors solely depend on the respective loads $\mathscr{T}(x), \mathscr{H}(x)$, and $\mathscr{D}(x)$, as well as the geometric parameters, $a$ and $h$. This phenomenon of decoupling and independence of material constants was also exhibited in the study of parallel constantly loaded periodic cracks in piezoelectric media by $\mathrm{Li}$ and Lee [29].

In contrast, however, if there is an interest in the phonon and phason displacement components and the electric potential in the vicinity of a crack in the stack, it is clear that if they are deduced using Equations (11) and (27)-(29), they will be heavily related additionally to the material moduli.

When all the loads applied to the stack of cracks are uniform, so that $F(x)=F=$ constant, the integral in Equation (43) can be calculated exactly and then $K_{F}^{S}$ reduces to

$$
K_{F}^{S}=\left\{\frac{h}{2 \pi} \tanh \left(\frac{\pi a}{h}\right)\right\}^{1 / 2} F .
$$

This is in agreement with the results of Li and Lee [29] for piezoelectric media.

Correspondingly, for a single uniformly loaded crack, the expression (44) can be calculated exactly as 


$$
\begin{aligned}
K_{T} & =\sqrt{\frac{a}{2}} T, \\
K_{H} & =\sqrt{\frac{a}{2}} H, \\
K_{D} & =\sqrt{\frac{a}{2}} D .
\end{aligned}
$$

Thus, with uniform loads $\mathscr{T}(x)=T=$ constant, $\mathscr{H}(x)=H=$ constant, and $\mathscr{D}(x)=D=$ constant on the stack of cracks,

$$
K_{F}^{S}=\left\{\frac{\tanh (\pi a / h)}{\pi a / h}\right\}^{1 / 2} K_{F},
$$

for $F=T, H$, and $D$, so in this situation each of the field intensity factors at a crack tip in the stack is of smaller magnitude than that near a corresponding single uniformly loaded crack. This so-called crack shielding behaviour is illustrated in Figure 3, where the variation with the relative spacing $h / a$ of the normalized field intensity factors, $K_{F}^{S} / K_{F}$, of a uniformly loaded stack is plotted. It is seen to occur decreasingly as $h / a$ increases from 0 to approximately 15 . This phenomenon was exhibited similarly, for example, in the numerical investigation of multiple cracks by Zhou et al. [30].

It is plain from Equations (27)-(29) that the analyses above would be invalid when $\bar{c}_{44} \bar{K}-\bar{R}^{2}=0$. But as yet, for one-dimensional hexagonal piezoelectric quasicrystals, the values presented of the moduli data are not entirely reliable. However, $\mathrm{Li}$ et al. [17] typically reported that $c_{44}=5.0 \times 10^{10} \mathrm{Nm}^{-2}, \quad R=1.2 \times 10^{9} \mathrm{Nm}^{-2}, \quad K=3.0 \times$ $10^{8} \mathrm{Nm}^{-2}, e_{15}=-0.138 \mathrm{Cm}^{-2}, e^{\prime}{ }_{15}=-0.160 \mathrm{Cm}^{-2}$, and $\varepsilon_{11}=82.6 \times 10^{-12} \mathrm{C}^{2} \mathrm{~N}^{-1} \mathrm{~m}^{-2}$. Then, clearly $\bar{c}_{44} \bar{K}-\bar{R}^{2}$ is nonzero, since the value of $\bar{c}_{44} \bar{K}$ is much greater than that of $\bar{R}^{2}$.

\section{Conclusions}

The fields of deformation generated around a stack of nonuniformly loaded parallel mode III shear cracks in onedimensional piezoelectric quasicrystals are studied by appropriately extending the technique of dislocation layers for simulating the cracks.

The system of three coupled singular integral equations which must be fulfilled by the density functions of the required dislocation arrays in order to satisfy the imposed boundary conditions is derived. These are neatly manipulated into singular integral equations that are amenable to solution exactly, using an appropriate explicit hyperbolic trigonometric function identity representation of the doubly-infinite series that are involved as summations of the contributions from all the cracks. Explicit expressions for the components of the phonon, phason, and electric fields are derived in a closed form, and their angular variations around a crack tip are deduced. Elegant representations for the field intensity factors are deduced.

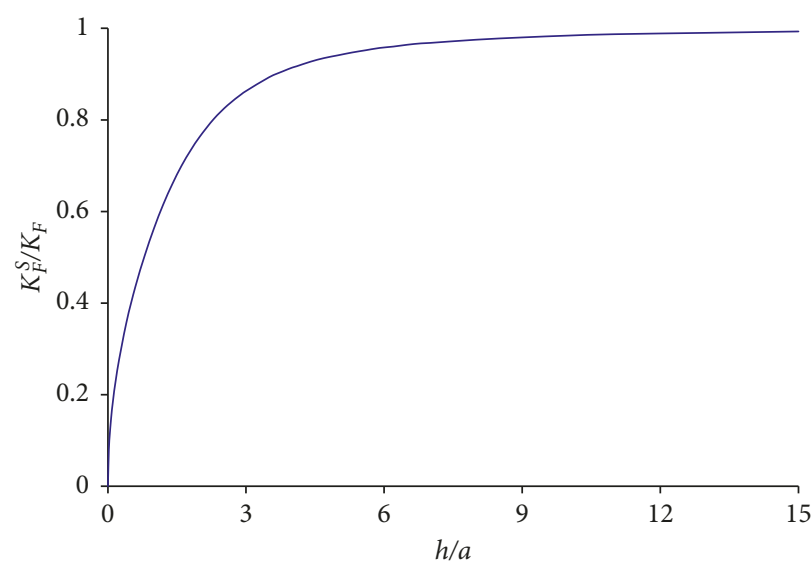

FIGURE 3: Variation of the normalized field intensity factors with the relative spacing in a stack of uniformly loaded cracks.

Even for a stack of uniformly loaded cracks, general representations such as these have not been presented before for any materials whatsoever. For example, in the special case of a stack of constantly loaded cracks in just piezoelectric media, only the field components along the crack lines, and thus the intensity factors, have been derived. Comparisons are drawn between the nonuniformly loaded and uniformly loaded situations.

It is demonstrated that the phenomenon of crack shielding occurs for small values of the relative spacing $h / a$, by illustrating graphically the variation of the ratio of the intensity factors for a stack of uniformly loaded cracks to those of a corresponding single crack.

As particular cases, of course, the previously unavailable corresponding simplified results for non-quasicrystals or non-piezoelectric or purely elastic media follow.

Representative numerical data are displayed graphically.

\section{Appendix}

The components $\sigma_{y z}$ and $\sigma_{x z}$ of the phonon stress are given by the expressions (30) and (31), respectively, for example, and involve integrals of the types

$$
\begin{aligned}
& I(x, y)=\int_{-a}^{a} \sum_{n=-\infty}^{\infty} \frac{x-x^{\prime \prime}}{\left(x-x^{\prime \prime}\right)^{2}+(y-n h)^{2}} r\left(x^{\prime \prime}\right) d x^{\prime \prime}, \\
& J(x, y)=-\int_{-a}^{a} \sum_{n=-\infty}^{\infty} \frac{y-n h}{\left(x-x^{\prime \prime}\right)^{2}+(y-n h)^{2}} r\left(x^{\prime \prime}\right) d x^{\prime \prime},
\end{aligned}
$$

for $r(x)=f(x), g(x)$, and $j(x)$.

It is particularly convenient to simultaneously calculate these by considering the complex combination $K(x, y)$ of them defined by 


$$
\begin{aligned}
K(x, y) & =I(x, y)+i J(x, y) \\
& =\int_{-a}^{a} \sum_{n=-\infty}^{\infty} \frac{1}{x-x^{\prime \prime}+i(y-n h)} r\left(x^{\prime \prime}\right) d x^{\prime \prime}, \\
K(x, y) & =\int_{-a_{2}}^{a_{2}} \frac{1}{\sinh \{\pi(x+i y) / h\}-x_{2}^{\prime \prime}} r\left(x_{2}^{\prime \prime}\right) d x_{2}^{\prime \prime} .
\end{aligned}
$$

In rewriting Equation (A.3) in the form (A.4), the doubly-infinite summation has been evaluated using the identities

$$
\begin{aligned}
\cot \pi z & =\frac{1}{\pi} \sum_{n=-\infty}^{\infty} \frac{1}{z-n}, \\
\cot (-i z) & =i \operatorname{coth} z,
\end{aligned}
$$

and the property that all the density functions are odd, in a manipulation and with a notation which is reminiscent of that in which the identity (23) was used to rewrite Equation (20) as Equation (26).

When the representations for $r_{2}\left(x_{2}^{\prime \prime}\right)$ in Equations (27)-(29) are incorporated into Equation (A.4), $K(x, y)$ is seen to involve relevant constant multiples of double integrals of the type:

$$
\begin{aligned}
L(x, y)= & \int_{-a_{2}}^{a_{2}}\left(a_{2}^{2}-x_{2}^{\prime \prime 2}\right)^{1 / 2} \\
& \int_{-a_{2}}^{a_{2}} \frac{1}{\left(a_{2}^{2}-x_{2}^{\prime \prime 2}\right)^{1 / 2}\left(x_{2}^{\prime}-x_{2}^{\prime \prime}\right)\left[\sinh \{\pi(x+i y) / h\}-x_{2}^{\prime \prime}\right]} d x_{2}^{\prime \prime} F\left(x_{2}^{\prime}\right) d x_{2}^{\prime},
\end{aligned}
$$

where $F(x)=\mathscr{T}(x), \mathscr{H}(x)$, or $\mathscr{D}(x)$. The inner integral in the above expression can be calculated using complex variable contour integration methods by applying Cauchy's residue theorem to evaluate the integral around a contour in the complex plane enclosing the singularities at $x_{2}^{\prime}$ and $\sinh \{\pi(x+i y) / h\}$ along the branch cuts from $-a_{2}$ to $a_{2}$ and a large circle around the origin to give

$$
L(x, y)=-\frac{i \pi e^{-i \theta}}{\mathscr{R}} \int_{-a_{2}}^{a_{2}} \frac{\left(a_{2}^{2}-x_{2}^{\prime 2}\right)^{1 / 2}}{\sinh \{\pi(x+i y) / h\}-x_{2}^{\prime}} F\left(x_{2}^{\prime}\right) d x_{2}^{\prime},
$$

with

$$
\mathscr{R} \mathrm{e}^{i \theta}=\left[a_{2}^{2}-\sinh ^{2}\left\{\frac{\pi(x+i y)}{h}\right\}^{1 / 2}\right]
$$

where the square root function's branches are chosen as in Equation (34). The real and imaginary parts of the representation (A.7) can then straightforwardly be deduced to give the integrals $I(x, y)$ and $J(x, y)$ in terms of a function $\mathscr{F}^{F}(\theta)$, defined for $F(x)=\mathscr{T}(x), \mathscr{H}(x)$, and $\mathscr{D}(x)$ by

$$
\mathscr{F}^{F}(\theta)=-\frac{1}{\pi \mathscr{R}} \int_{-a_{2}}^{a_{2}} \frac{\mathscr{M}_{1}\left(x, x_{2}^{\prime}, \theta\right)}{\mathscr{M}_{2}\left(x, x_{2}^{\prime}\right)}\left(a_{2}^{2}-x_{2}^{\prime 2}\right)^{1 / 2} F\left(x_{2}^{\prime}\right) d x_{2}^{\prime},
$$

where

$$
\begin{aligned}
\mathscr{M}_{1}\left(x, x_{2}^{\prime}, \theta\right)= & \left\{\sinh \left(\frac{\pi x}{h}\right) \cos \left(\frac{\pi y}{h}\right)-x_{2}^{\prime}\right\} \sin \theta \\
& +\cosh \left(\frac{\pi x}{h}\right) \sin \left(\frac{\pi y}{h}\right) \cos \theta, \\
\mathscr{M}_{2}\left(x, x_{2}^{\prime}\right)= & \left\{\sinh \left(\frac{\pi x}{h}\right) \cos \left(\frac{\pi y}{h}\right)-x_{2}^{\prime}\right\}^{2} \\
& +\cosh ^{2}\left(\frac{\pi x}{h}\right) \sin ^{2}\left(\frac{\pi y}{h}\right) .
\end{aligned}
$$

If preferred, alternatively, partial fractions, complex conjugate functions, and contour integration methods can used to calculate $I(x, y)$ and $J(x, y)$.

\section{Data Availability}

The data used to support the findings of this study are available from the corresponding author upon request.

\section{Conflicts of Interest}

The author declares that there are no conflicts of interest regarding the publication of this article.

\section{References}

[1] A. A. Griffith, "The phenomena of rupture and flow in solids," Philosophical Transactions of the Royal Society A: Mathematical, Physical and Engineering Sciences, vol. 221, no. 582-593, pp. 163-198, 1920.

[2] I. N. Sneddon and M. Lowengrub, Crack Problems in the Classical Theory of Elasticity, John Wiley and Sons, New York, NY, USA, 1969.

[3] D. Shechtman, I. Blech, D. Gratias, and J. W. Cahn, "Metallic phase with long-range orientational order and no translational symmetry," Physical Review Letters, vol. 53, no. 20, pp. 1951-1953, 1984.

[4] X.-F. Li, T.-Y. Fan, and Y.-F. Sun, “A decagonal quasicrystal with a Griffith crack," Philosophical Magazine A, vol. 79, no. 8, pp. 1943-1952, 1999.

[5] X.-F. Li, "A general solution of elasto-hydrodynamics of twodimensional quasicrystals," Philosophical Magazine Letters, vol. 91, no. 4, pp. 313-320, 2011.

[6] X. F. Li, "Elastohydrodynamic problems in quasicrystal elasticity theory and wave propagation," Philosophical Magazine, vol. 93, no. 13, pp. 1500-1519, 2013.

[7] D. H. Ding, W. G. Yang, C. Z. Hu, and R. H. Wang, "Generalized elasticity theory of quasicrystals," Physical Review B, vol. 48, no. 10, pp. 7003-7010, 1993.

[8] J. H. Guo, J. Yu, and Y. M. Xing, "Anti-plane analysis on a finite crack in a one-dimensional hexagonal quasicrystal strip," Mechanics Research Communications, vol. 52, pp. 4045, 2013.

[9] T.-Y. Fan, Z.-Y. Tang, and W.-Q. Chen, "Theory of linear, nonlinear and dynamic fracture for quasicrystals," Engineering Fracture Mechanics, vol. 82, pp. 185-194, 2012.

[10] T. Y. Fan, The Mathematical Theory of Elasticity of Quasicrystals and Its Applications, Science Press, Springer-Verlag, Beijing, China, 2016. 
[11] T. Y. Fan, "Mathematical theory and methods of mechanics of quasicrystalline materials," Engineering, vol. 5, no. 4, pp. 407-448, 2013.

[12] T. P. Yadav, "Recent research and development on quasicrystals," AIMS Materials Science, vol. 4, no. 1, pp. 172-177, 2017.

[13] G. Altay and M. C. Dökmeci, "On the fundamental equations of piezoelectricity of quasicrystals," International Journal of Solids and Structures, vol. 49, no. 23-24, pp. 3255-3262, 2012.

[14] C.-L. Li and Y.-Y. Liu, "The physical property tensors of onedimensional quasicrystals," Chinese Physics, vol. 13, no. 6, pp. 924-931, 2004.

[15] X. Wang and E. Pan, "Analytical solutions for some defect problems in 1D hexagonal and 2D octagonal quasicrystals," Pramana-Journal of Physics, vol. 70, no. 5, pp. 911-933, 2008.

[16] L.-Z. Yang, Y. Gao, E. Pan, and N. Waksmanski, "Electricelastic field induced by a straight dislocation in onedimensional quasicrystals," Acta Physica Polonica A, vol. 126, no. 2, pp. 467-470, 2014

[17] X. Y. Li, P. D. Li, T. H. Wu, M. X. Shi, and Z. W. Zhu, “Threedimensional fundamental solutions for one-dimensional hexagonal quasicrystal with piezoelectric effect," Physics Letters A, vol. 378, no. 10, pp. 826-834, 2014.

[18] J. Yu, J. Guo, E. Pan, and Y. Xing, "General solutions of plane problem in one-dimensional quasicrystal piezoelectric materials and its application on fracture mechanics," Applied Mathematics and Mechanics, vol. 36, no. 6, pp. 793-814, 2015.

[19] J. Yu, J. Guo, and Y. Xing, "Complex variable method for an anti-plane elliptical cavity of one-dimensional hexagonal piezoelectric quasicrystals," Chinese Journal of Aeronautics, vol. 28, no. 4, pp. 1287-1295, 2015.

[20] L. Zhang, D. Wu, W. Xu et al., "Green's functions of onedimensional quasicrystal bi-material with piezoelectric effect," Physics Letters A, vol. 380, no. 39, pp. 3222-3228, 2016.

[21] J. Guo and E. Pan, "Three-phase cylinder model of onedimensional piezoelectric quasi-crystal composites," Journal of Applied Mechanics, vol. 83, no. 8, article 081007, 2016.

[22] C. Fan, Y. Li, G. Xu, and M. Zhao, "Fundamental solutions and analysis of three-dimensional cracks in one-dimensional hexagonal piezoelectric quasicrystals," Mechanics Research Communications, vol. 74, pp. 39-44, 2016.

[23] J. Yang and X. Li, "Analytical solutions of problem about a circular hole with a straight crack in one-dimensional hexagonal quasicrystals with piezoelectric effects," Theoretical and Applied Fracture Mechanics, vol. 82, pp. 17-24, 2016.

[24] J. Guo, Z. Zhang, and Y. Xing, "Antiplane analysis for an elliptical inclusion in 1D hexagonal piezoelectric quasicrystal composites," Philosophical Magazine, vol. 96, no. 4, pp. 349-369, 2016.

[25] G. E. Tupholme, "One-dimensional piezoelectric quasicrystals with an embedded moving, non-uniformly loaded shear crack," Acta Mechanica, vol. 228, no. 2, pp. 547-560, 2017.

[26] G. E. Tupholme, "A non-uniformly loaded anti-plane crack embedded in a half-space of a one-dimensional piezoelectric quasicrystal," Meccanica, vol. 53, no. 4-5, pp. 973-983, 2018.

[27] Y.-B. Zhou and X.-F. Li, "Two collinear mode-III cracks in one-dimensional hexagonal piezoelectric quasicrystal strip," Engineering Fracture Mechanics, vol. 189, pp. 133-147, 2018.

[28] J.-C. Han and B.-L. Wang, "Electromechanical model of periodic cracks in piezoelectric materials," Mechanics of Materials, vol. 37, no. 12, pp. 1180-1197, 2005.

[29] X.-F. Li and K. Y. Lee, "A piezoelectric material with a periodic distribution of slant mode-III cracks," Mechanics of Materials, vol. 37, no. 1, pp. 189-200, 2005.
[30] Z.-G. Zhou, P.-W. Zhang, and G. Li, "Interactions of multiple parallel symmetric permeable mode-III cracks in a piezoelectric material plane," European Journal of Mechanics-A/ Solids, vol. 28, no. 4, pp. 728-737, 2009.

[31] M. M. Monfared and M. Ayatollahi, "Interactions of multiple cracks in a transversely isotropic piezoelectric plane under mixed mode condition," Engineering Fracture Mechanics, vol. 180, pp. 87-104, 2017.

[32] B. A. Bilby and J. D. Eshelby, "Dislocations and the theory of fracture," in Fracture, H. Liebowitz, Ed., vol. 1, pp. 99-182, Academic Press, New York, NY, USA, 1968.

[33] R. W. Lardner, Mathematical Theory of Dislocations and Fracture, University of Toronto Press, Toronto, ON, Canada, 1974.

[34] N. I. Muskhelishvili, Singular Integral Equations, Noordhoff International Publishing, Leyden, Netherlands, 1953.

[35] F. D. Gakhov, Boundary Value Problems, Pergamon, Oxford, UK, 1966. 


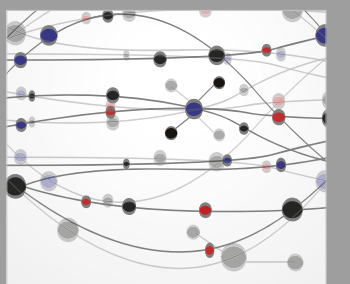

The Scientific World Journal
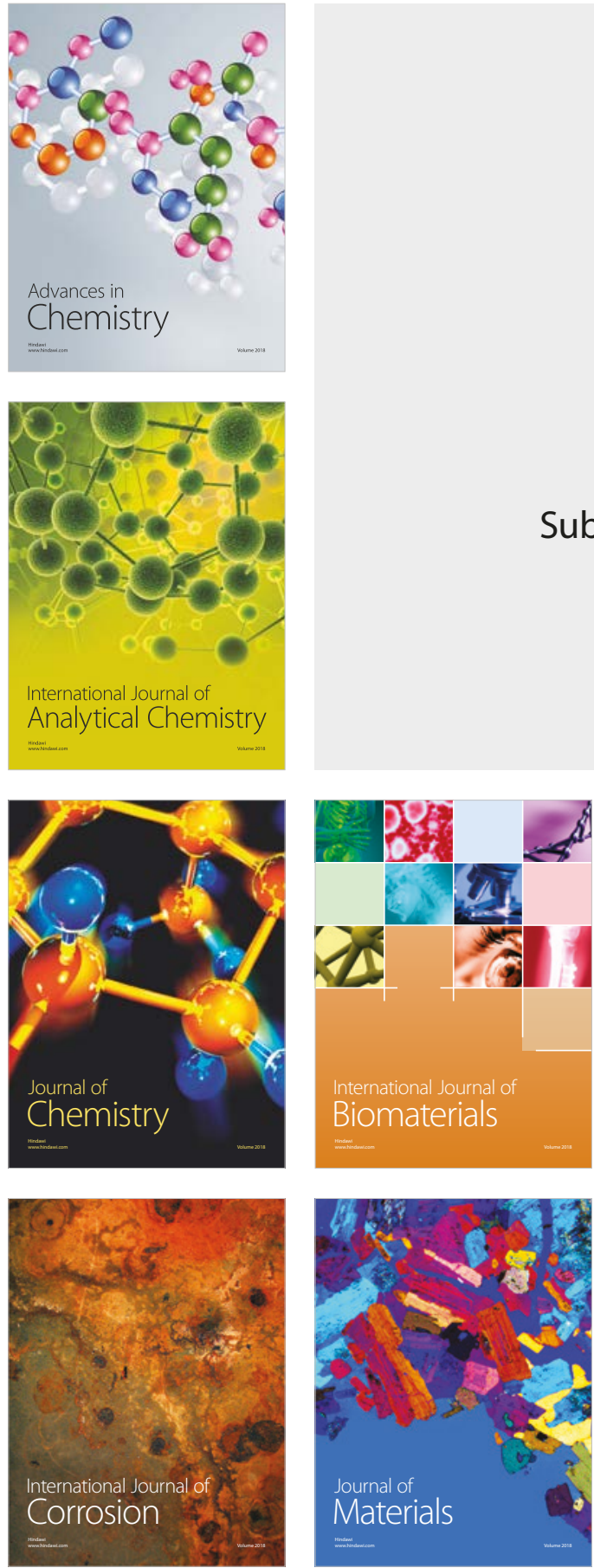

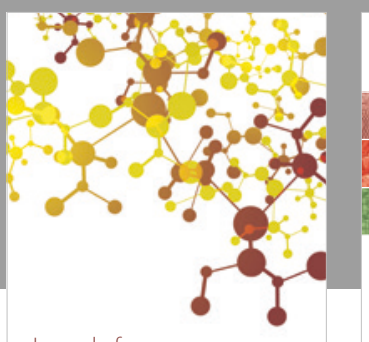

Journal of

Applied Chemistry
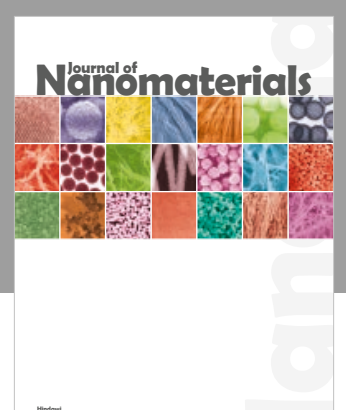

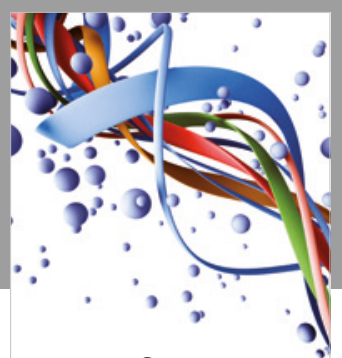

Scientifica

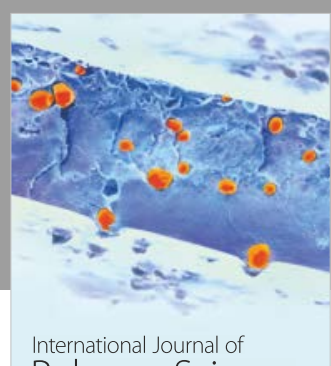

Polymer Science

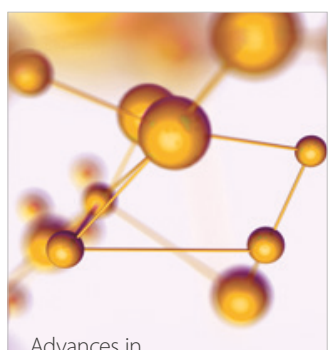

Physical Chemistry
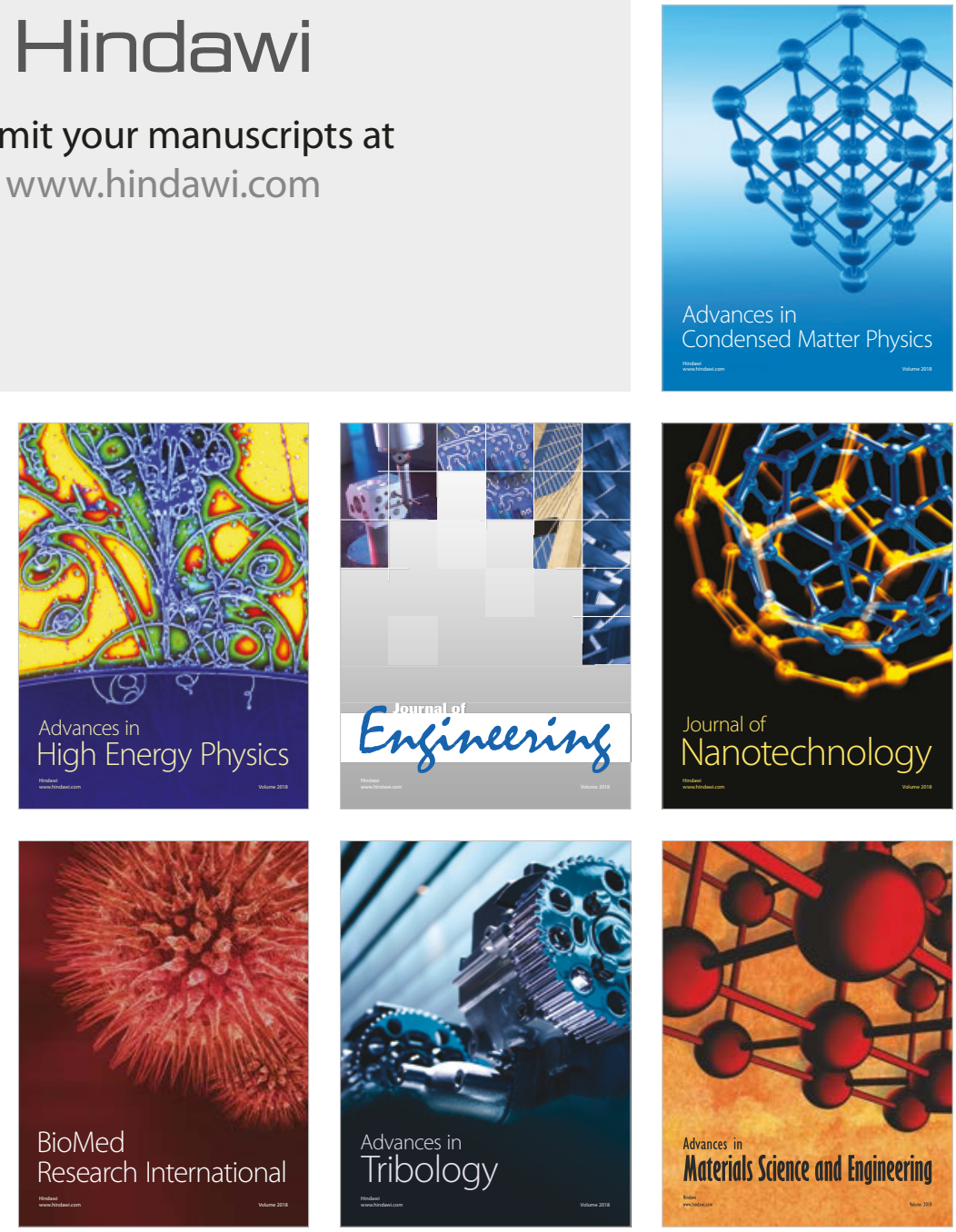